\title{
The effect of intra-articular hyaluronate and tramadol injection on patients with adhesive capsulitis of the shoulder
}

\author{
Kyung-Hee Kim ${ }^{\mathrm{a}}$, Jung-Woo Suh ${ }^{\mathrm{b}}$ and Ki Young $\mathrm{Oh}^{\mathrm{b}, *}$ \\ ${ }^{a}$ Department of Laboratory Medicine, Gachon University Gil Medical Center, Korea \\ ${ }^{\mathrm{b}}$ Department of Physical Medicine and Rehabilitation, Soonchunhyang University Cheonan Hospital, Korea
}

\begin{abstract}
.
BACKGROUND: Local administration of opioids causes effective analgesia without adverse effects related to the central nervous system. After the beneficial demonstration of peripheral opioid receptors in joint synovia, intra-articular opioid injections were used for pain treatment. Clinical studies have reported the safety and efficacy of hyaluronate injection in the shoulder joint of patients with osteoarthritis, periarthritis, rotator cuff tears, and adhesive capsulitis.

OBJECTIVES: To estimate the efficacy of intra-articular hyaluronate and tramadol injection for adhesive capsulitis of the shoulder compared with that of intra-articular hyaluronate injection alone.

METHODS: Thirty patients with adhesive capsulitis of the shoulder were randomized to the hyaluronate group $(n=16)$ or the tramadol group $(n=14)$. Hyaluronate group members were administered five weekly intra-articular hyaluronate injections; tramadol group members were administered three weekly intra-articular hyaluronate and tramadol injections and then two weekly intra-articular injections of hyaluronate. Visual Analog Scale (VAS), passive range of motion (PROM) of the shoulder joint, and Shoulder Pain and Disability Index (SPADI) scores were assessed at baseline and weeks 1, 2, 3, 4, and 6 after the initial injection. RESULTS: A significant improvement was observed in VAS, PROM, and SPADI scores between time points in both groups. In comparison in both groups at weeks 1 and 2 after the initial injection the VAS scores of the tramadol group were significantly lower than those of the hyaluronate group.

CONCLUSIONS: Intra-articular hyaluronate with tramadol showed more rapid and strong analgesic effects than intra-articular hyaluronate alone and did not induce any adverse effects.
\end{abstract}

Keywords: Adhesive capsulitis, analgesia, intraarticular injection, opioids

\section{Introduction}

Adhesive capsulitis of the shoulder is a condition commonly encountered in outpatient clinics It is thought to have an incidence of $3 \%-5 \%$ in the general population and up to $20 \%$ in those with diabetes [1]. In 1934, Codman described it as a disease with a slow onset; patients have pain near the insertion of the deltoid muscle, cannot sleep on the affected side, and

\footnotetext{
*Corresponding author: Ki Young Oh, Soonchunhyang University Cheonan Hospital, Bongmyeong-dong, Dongnam-gu, Cheonansi, Chungcheongnam-do 330-721, Korea. Tel.: +82 41570 3884; Fax: +82 41570 2776; E-mail: okokykkh@schmc.ac.kr.
}

show limited flexion and external rotation of the shoulder joints, but they appear normal on radiographs [2]. Recently, the American Academy of Orthopedic Surgeons defined adhesive capsulitis of the shoulder as a "disease characterized by progressive limitation in the active and passive ranges of motion (AROM and PROM) of shoulder joints that does not show any abnormality on radiographs except signs of osteoporosis" [3].

The natural history of adhesive capsulitis remains a matter of debate. Some have suggested that adhesive capsulitis is self-limiting and does not need to be treated [4]. According to studies that conducted follow-up observations, one reported that $96-100 \%$ of patients showed normal shoulder joint functions 2 
and 4 years later [5]. In contrast, another study indicated that $50 \%$ of patients reported pain and stiffness 7 years later and that functional limitations were observed in $11 \%$ [6]. Therefore, various treatments are being used to decrease pain and increase ROM more quickly than does spontaneous recovery. However, the treatment outcomes are not always satisfactory [7]. Non-surgical treatments of adhesive capsulitis of the shoulder include physical therapy, pharmacological treatment, intra-articular injection, and shoulder joint capsule elongation exercise treatments that aim to decrease the pain and inflammation while recovering ROM and function of the joints; surgical treatments include manual therapy performed under general anesthesia, synovectomy, and arthroscopic capsular release [8]. Typical drugs that are used for intraarticular injection are hyaluronate and steroids, and treatment using opioids has also been reported [9].

Injection of hyaluronate and steroids into the shoulder joints began because of the belief that inflammatory responses are the major cause of adhesive capsulitis of the shoulder and that anti-inflammatory treatments are required to improve the symptoms and prevent the progression of fibrosis of the capsule because inflammation plays an important role in fibrosis [10].

Intra-articular injection of hyaluronate into the shoulder joints has been confirmed to be effective for pain reduction, enhancement of ROM, antiinflammatory actions, protection of cartilage, and improvement of synovial fluid indices, and it is being used as a safe and effective treatment for adhesive capsulitis of the shoulder [11].

Intra-articular injection of a steroid alone has shown satisfactory results for treating adhesive capsulitis of the shoulder [12]. Although injection of hyaluronate alone has also resulted in significant improvements in pain, ROM of the shoulder joints, and evaluation of shoulder joint functions when compared to the control group, the effects are known to appear approximately 2 weeks later [13].

According to studies, hyaluronate and steroid do not differ significantly in terms of treatment effects, and the combined use of hyaluronate and steroid has been reported to result in better improvement of ROM, including external and internal rotation, of the joints [4, $9,14,15]$.

Despite the findings that combined therapy has more significant effects, one should watch for deleterious effects of the steroid such as peri-articular calcifications, tendinopathy, avascular necrosis, acute synovitis exerted on surrounding ligaments and cartilage and general side effects [16-18]. Therefore, in this study, we combined drugs other than steroids to obtain better treatment effects for adhesive capsulitis of the shoulder.

Since the existence of opioid receptors in peripheral tissues, including inside articular cavities, has been proven immunologically and functionally, intraarticular injection of opioids has been performed to decrease articular pain [13,19]. Joshi et al. reported that articular pain was successfully controlled using morphine after manual therapy of adhesive capsulitis of the shoulder [9]. Regarding pain occurring after shoulder joint surgery, although some controversy remains, studies have reported that injections were effective for controlling the pain [20-22].

Since the finding that opioid receptors also exist in peripheral systems outside the central nervous system (CNS), especially in synovia inside inflamed articular cavities, local opioid injections, including intraarticular injections, have been administered to control pain without any risk of CNS-related adverse effects $[11,12]$.

Although opioids show strong analgesic effects by activating the receptors in the CNS, they can lead to CNS-related adverse effects such as nausea, vomiting, dizziness, and respiratory depression [22,23].

There are three types of peripheral opioid receptors (mu, delta, and kappa). Although they are not observed in normal tissues, they are formed by inflammation, and the receptor activity increases 400- to 500fold once the inflammatory phase begins. If opioids are locally administered to inflamed tissues, then opioids bind peripheral receptors to increase the potassium current and to decrease the calcium current in the cell body of sensory neurons. By doing so, opioids suppress the excitation of nerve fibers and release of neurotransmitters, thereby showing a strong analgesic effect in inflamed tissues [24,25].

Tramadol is an opioid that is a weak mu receptor agonist. The activation of mu receptors accounts for $40 \%$ of its analgesic mechanism, and the other $60 \%$ is accounted for by the activation of $\alpha-2$ (alpha-2) receptors and serotonergic receptors in the antinociceptive system [26,27]. Because the expression of adrenergic and serotonergic receptors is not adequate in inflamed tissues, analgesic potency of tramadol is weaker than that of morphine; however, the analgesic effect of tramadol lasts relatively long [28]. Therefore, we expected that combining hyaluronate with tramadol, which shows a long-lasting analgesic effect, would be a better treatment than the combination of hyaluronate with mor- 
Table 1

Characteristics of study subjects

\begin{tabular}{lcc}
\hline & $\begin{array}{c}\text { Hyaluronate } \\
\text { group }\end{array}$ & $\begin{array}{c}\text { Hyaluronate plus } \\
\text { tramadol group }\end{array}$ \\
\hline N of subjects & 16 & 14 \\
Age & $54.6 \pm 5.8$ & $55.9 \pm 5.8$ \\
Gender (male/female) & $11 / 5$ & $9 / 5$ \\
Affected side (right/left) & $10 / 6$ & $9 / 5$ \\
Duration of symptom (months) & $3.9 \pm 1.8$ & $4.0 \pm 1.6$ \\
Baseline VAS & $7.3 \pm 1.2$ & $7.0 \pm 1.5$ \\
Baseline ROM & & \\
$\quad$ Flexion & $141.4 \pm 19.7$ & $140.1 \pm 19.7$ \\
$\quad$ Abduction & $129.4 \pm 23.3$ & $130.4 \pm 24.9$ \\
$\quad$ External rotation & $55.0 \pm 15.1$ & $55.2 \pm 16.2$ \\
$\quad$ Internal rotation & $53.8 \pm 17.3$ & $52.3 \pm 16.9$ \\
Baseline SPADI & $70.3 \pm 18.7$ & $71.6 \pm 17.5$ \\
\hline
\end{tabular}

Mean \pm SD. SD, standard deviation.

phine, which is used for short-term analgesia after surgery Although multiple recent clinical studies have shown that tramadol is effective for alleviating postoperative pain when injected into knee joints and temporomandibular joints, there has been no report of tramadol used in shoulder joints [29-32].

This study aimed to investigate whether significant changes occur in pain, ROM, and function of shoulder joints when hyaluronate and tramadol are injected together into the joints as treatment for adhesive capsulitis of the shoulder.

The mean age of the 16 patients in the hyaluronate group was 54.6 years, and they had been experiencing symptoms for $3.9 \pm 1.8$ months. There were 11 males and five females; the right shoulder was affected in 10 patients and the left shoulder was affected in six patients. The mean age of the 14 patients in the tramadol group was 55.9 years, and they had been experiencing symptoms for $4.0 \pm 1.6$ months. There were nine males and five females; the right shoulder was affected in nine patients and the left shoulder was affected in five patients. There was no statistically significant difference in the general characteristics of the two groups (Table 1).

\section{Methods}

Among patients who visited the Rehabilitation Department Outpatient Clinic at Soonchunhyang University Hospital because of pain and limited movement of the shoulder joints, we began investigating 40 patients (sample size was calculated using G-power 3.1 statistic software alpha error $=0.05$, power $=0.8$ ) whose adhesive capsulitis of the shoulder was confirmed based on medical history, physical examina-

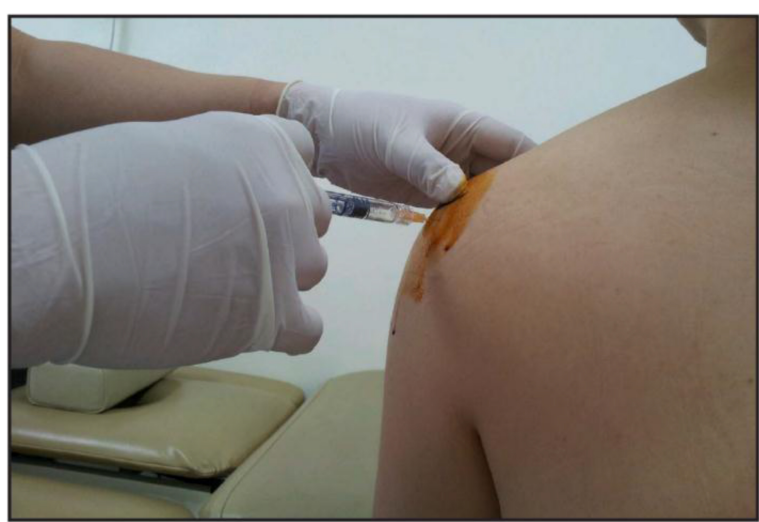

Fig. 1. Posterior technique for shoulder intra-articular injection.

tion, simple X-ray methods, and musculoskeletal ultrasonography. However, 10 patients dropped out of the study (they discontinued study participation of their own accord); therefore, a total of 30 patients were included. Approval was granted by the Institutional Review Board of Soonchunhyang University Hospital (Trial registration no. ISRCTN13453452) and written informed consent was obtained from each patient. Patients were identified only by number, not by name or initials. Adhesive capsulitis of the shoulder was diagnosed when PROM of the affected shoulder joint was limited in at least two directions (anterior flexion, external rotation, and internal rotation) by more than $25 \%$ or 30 degrees when compared to the healthy shoulder joint or normal PROM and when there was accompanying shoulder joint pain. Patients were excluded if they had trauma, stroke, endocrine diseases, or arthritis in the shoulder, if they had been administered an MAO (monoamine oxidase) inhibitor during the past 2 weeks, if they had hypersensitivity to opioids, if they had received an intra-articular injection in the shoulder during the past 6 months, if they had undergone other surgeries, if they had been experiencing symptoms for more than 9 months, or if ultrasonography showed a full-thickness tear in some tendons of the rotator cuff. All patients were educated by doctors regarding self-performed exercise treatment methods and received printouts to aid them in conducting shoulder joint capsule elongation exercises at least twice daily No physical therapy was performed.

The patients were randomly allocated into two groups. Randomization was conducted in blocks of ten (each manila envelope contained 5 papers from the hyaluronate group and 5 from the tramadol group) The control group (hyaluronate group) was injected only with hyaluronate (Hyal Shin Poong PhD, Korea, 


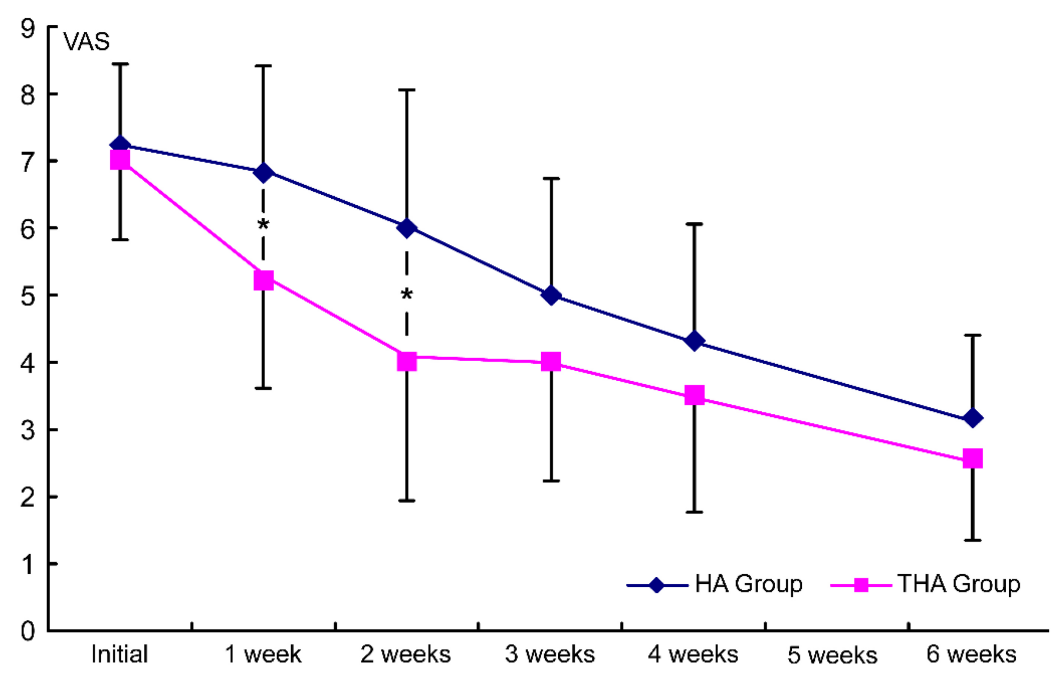

Fig. 2. Intergroup comparison of the injection effects on VAS. HA group, hyaluronate group; THA group, hyaluronate plus tramadol group.

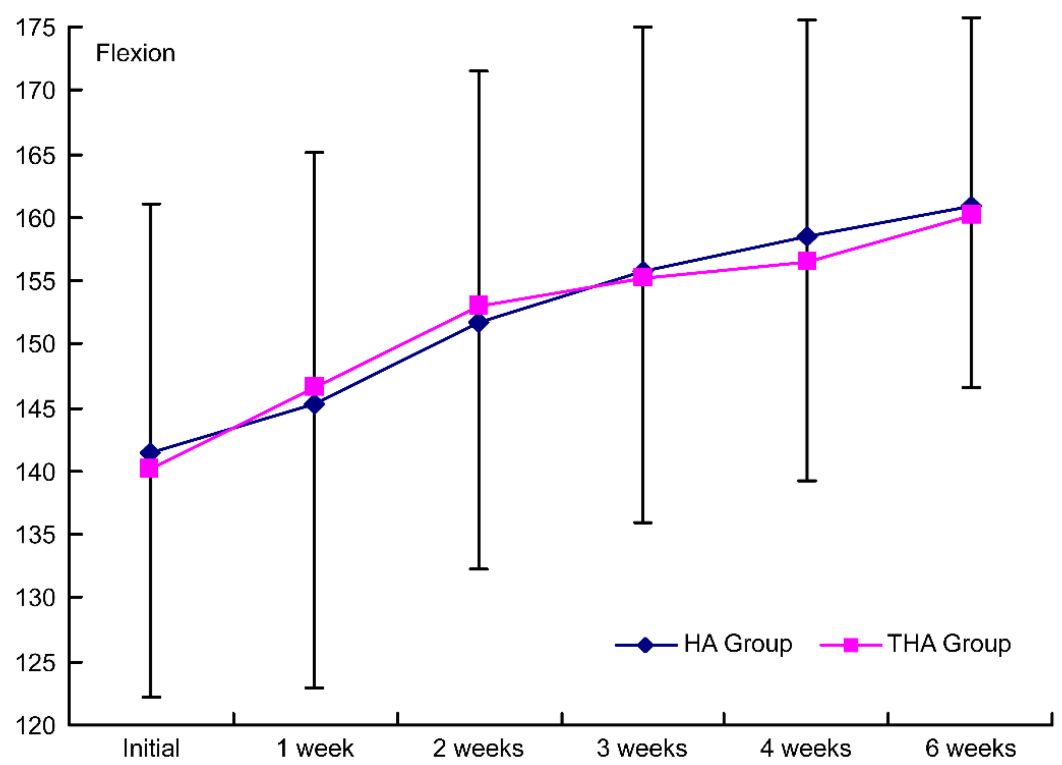

Fig. 3. Intergroup comparison of the injection effects on flexion. HA group, hyaluronate group; THA group, hyaluronate plus tramadol group.

$2.5 \mathrm{~mL}$ injection, $1 \%$ hyaluronan, molecular weight $940-1,020 \mathrm{kDa})$ five times in 1 week intervals; the experimental group (tramadol group) received hyaluronate and tramadol (Tridol Shin Poong $\mathrm{PhD}$, Korea, $100 \mathrm{mg}, 1 \mathrm{~mL}$ injection) $50 \mathrm{mg}$ injections three times, and then they received two injections of only hyaluronate afterward. Intra-articular injection was administered by one doctor using the posterior approach (Fig. 1). The patient sat on a chair and the affected shoulder was adducted and internally rotated. Afterward, the doctor positioned his/her left index finger on the patient's coracoid process tip and his/her left thumb on the patient's acromion angle. A $23 \mathrm{G}$ syringe needle was directed at the index finger and inserted below the thumb until reaching the joint capsule; each drug was injected afterward.

The VAS was used to measure the degree of pain. To measure PROM, we followed the recommendations of the American Academy of Orthopedic Surgeons and measured abduction on the frontal plane, anterior flexion at 0 degrees of adduction, and external and internal rotation when the elbow joint was flexed at 90 degrees using a goniometer. To measure dysfunction of the shoulder joints, we used SPADI to measure five 


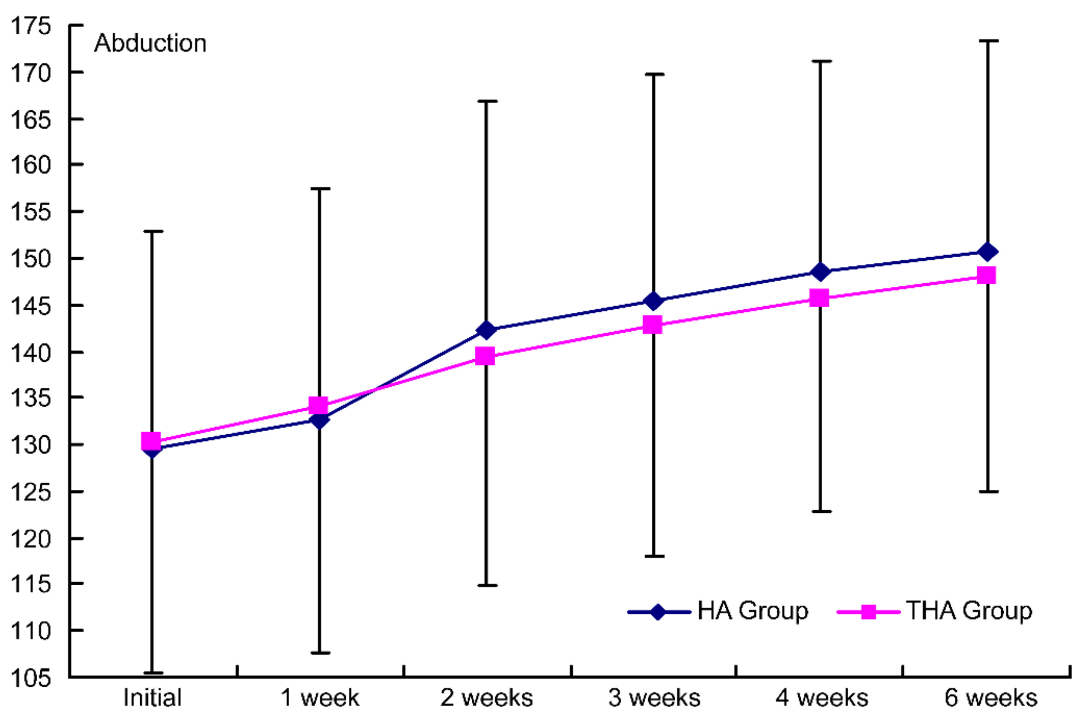

Fig. 4. Intergroup comparison of the injection effects on abduction. HA group, hyaluronate group; THA group, hyaluronate plus tramadol group.

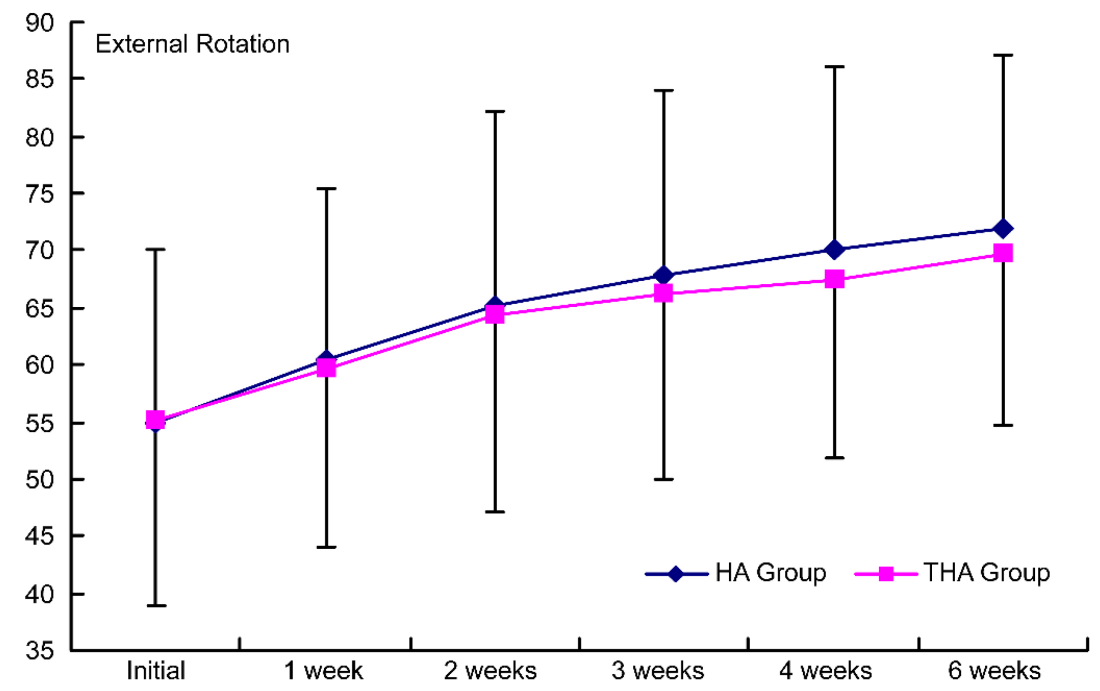

Fig. 5. Intergroup comparison of the effects on external rotation by the injection. HA group, hyaluronate group; THA group, hyaluronate plus tramadol group.

items regarding pain and eight items regarding disability; the total score was out of 100 . To measure the treatment effects, VAS, PROM, and SPADI were measured by another doctor before the treatments and at 1,2,3, 4 , and 6 weeks after the treatments.

SPSS version 17.0 was used for statistical analysis. Independent t-tests were conducted to compare the general characteristics of the groups, whereas repeated measures analysis of variance (ANOVA) was used to compare the time-dependent treatment effects observed in the groups. The significance level was set at $P<0.05$.

\section{Results}

\subsection{Change in VAS in each group after treatment}

Before the injection treatments, the mean VAS of the hyaluronate group was $7.3 \pm 1.2$ whereas that of the tramadol group was $7.0 \pm 1.5$. VAS decreased significantly with time in both groups $(P<0.05)$. VAS measured at the first and second visits after the initial treatment decreased more significantly in the tramadol group than in the hyaluronate group $(P<0.05)$ (Fig. 2). 


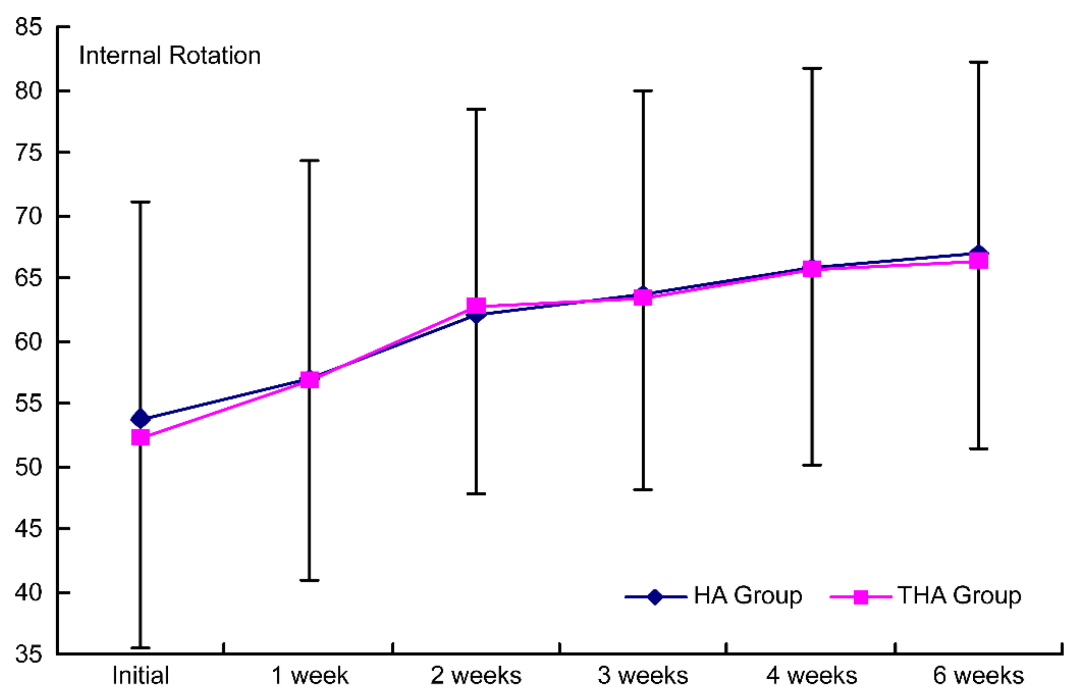

Fig. 6. Intergroup comparison of the effects on internal rotation by the injection. Group 1, control group; group 2, experimental group; HA group, hyaluronate group; THA group, hyaluronate plus tramadol group.

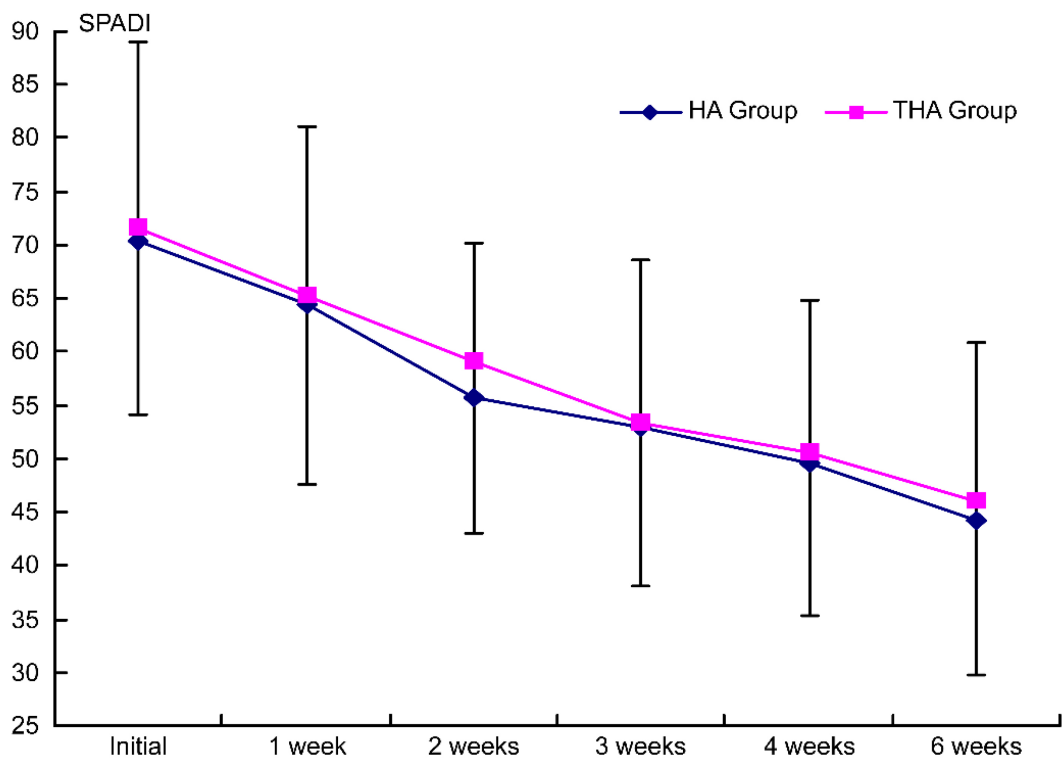

Fig. 7. Intergroup comparison of the effects on SPADI scores by injection. HA group, hyaluronate group; THA group, hyaluronate plus tramadol group.

\subsection{Change in PROM of each group after treatment}

PROM increased significantly with time in both groups $(P<0.05)$ No significant difference was observed between the groups (Figs 3-6).

\subsection{Change in SPADI score of each group after treatment}

SPADI scores decreased significantly with time in both groups $(P<0.05)$. There was no significant difference between the groups (Fig. ??).

\section{Discussion}

In this study, the group that received the combined injection with tramadol showed more significant improvements in VAS at 1 and 2 weeks after treatment; therefore, the analgesic effect of tramadol seems to be 
significant. Moreover tramadol showed more immediate analgesic effects than hyaluronate alone.

Alagol et al. and Joshi et al. ruled out effects resulting from systemic absorption based on the facts that tramadol and morphine, when each was injected intraarticular, did not induce many systemic side effects and the plasma concentrations did not show any significant increase $[29,33]$. Although we did not measure the plasma concentrations, there were no side effects in our study; therefore, the analgesic effect does not seem to have been due to systemic absorption.

ROM of the shoulder joints improved in both groups without any significant inter-group difference. Although hyaluronate has been found to increase ROM, no such effect of tramadol has been reported yet; moreover, as shown by O'Kane and Jorma et al., self-performed exercise treatment can also improve ROM [34,35]. Because we injected hyaluronate or tramadol and because the patients also completed selfperformed exercise treatment, the two groups would have shown sufficient treatment effects without any significant inter-group difference.

SPADI is usually used to evaluate dysfunction of the shoulder joints and consists of five items assessing pain and eight items assessing disability. Here, pain refers to the pain that appears when performing functional movements, and it is influenced by ROM of the shoulder joints. Therefore, SPADI scores would not have shown any significant difference between the groups in contrast to VAS, which improved more significantly in the tramadol group.

The limitations of this study include the relatively small sample size and short follow-up. Moreover, because we administered intra-articular injections using a blind method rather than the ultrasonography-assisted method, the accuracy could have been compromised. In addition, we did not evaluate how well the patients completed the self-performed exercise treatment. Future studies need to be conducted using larger samples need to increase the accuracy of injections through the ultrasonography-assisted method, and need to be conducted with patients with chronic pain that has entered the fibrosis phase past the inflammatory phase. Furthermore, longer-term effects need to be investigated.

\section{Conclusions}

In conclusion, intra-articular injection of hyaluronate and tramadol, when used as a treatment for adhesive capsulitis of the shoulder, showed an earlier response than hyaluronate injection alone. Moreover, to increase ROM of the shoulder joint concurrent exercise treatment should be used for more favorable outcomes.

\section{Acknowledgments}

This work was supported by the Soonchunhyang University Research Fund.

We would like to thank Editage (www.editage.co.kr) for English language editing.

\section{Conflict of interest}

The authors have no conflicts of interest to declare.

\section{References}

[1] Manske RC, Prohaska D. Diagnosis and management of adhesive capsulitis. Curr Rev Musculoskelet Med 2008; 1: 180189.

[2] Codman EA. Tendinitis of the short rotators. In: Codman EA, ed. The Shoulder: Rupture of the Supraspinatus Tendon and Other Lesions in or About Subacromial Bursa. Boston: Thomas Todd Co.; 1934: pp. 78.

[3] Griesser MJ, Harris JD, Campbell JE, et al. Adhesive capsulitis of the shoulder: a systematic review of the effectiveness of intra-articular corticosteroid injections. J Bone Joint Surg Am 2011; 93: 1727-1733.

[4] Neviaser AS, Neviaser RJ. Adhesive capsulitis of the shoulder. Am Acad Orthop Surg 2011; 19(9): 536542.

[5] Grey RG. The natural history of "idiopathic" frozen shoulder. J Bone Joint Surg Am 1978; 60: 564.

[6] Brue S, Valentin A, Forssblad M, et al. Idiopathic adhesive capsulitis of the shoulder: a review. Knee Surg Sports Traumatol Arthrosc 2007; 15: 1048-1054.

[7] Harris JD, Griesser MJ Copelan A, et al. Treatment of adhesive capsulitis with intra-articular hyaluronate: A systematic review. Int J Shoulder Surg 2011; 5: 31-37.

[8] Neviaser AS, Hannafin JA. Adhesive capsulitis: a review of current treatment. Am J Sports Med 2010; 38: 2346-2356.

[9] Joshi GP, McCarroll SM. Intra-articular morphine for the management of frozen shoulder. Anaesthesia 1992; 47: 627.

[10] Lee HJ, Lim KB, Kim DY, et al. Randomized controlled tria for efficacy of intra-articular injection for adhesive capsulitis: ultrasonography-guided versus blind technique. Arch Phys Med Rehabil 2009; 90: 1997-2002.

[11] Iwata H. Pharmacologic and clinical aspects of intraarticular injection of hyaluronate. Clin Orthop Relat Res 1993; 289: 285-291.

[12] Lorbach O, Anagnostakos K, Scherf C, et al. Nonoperative management of adhesive capsulitis of the shoulder: oral cortisone application versus intra-articular cortisone injections. $J$ Shoulder Elbow Surg 2010; 19: 172-179. 
[13] Bartho L Stein C, Herz A. Involvement of capsaicin-sensitive neurones in hyperalgesia and enhanced opioid antinociception in inflammation. Naunyn Schmiedebergs Arch Pharmacol 1990; 342: 666-670.

[14] Calis M, Demir H, Ulker S, et al. Is intraarticular sodium hyaluronate injection an alternative treatment in patients with adhesive capsulitis? Rheumatol Int 2006; 26: 536-540.

[15] Rovetta G, Monteforte P. Intraarticular injection of sodium hyaluronate plus steroid versus steroid in adhesive capsulitis of the shoulder. Int J Tissue React 1998; 20: 125-130.

[16] Cho KH, Lee H, Kim JS, et al. The effect of subacromial bursa injection of hyaluronate in patients with adhesive capsulitis of shoulder joint. J Korean Acad Rehabil Med 2002; 26: 73-80.

[17] Paavola M, Kannus P, Jarvinen TA, et al. Treatment of tendon disorders. Is there a role for corticosteroid injection? Foot Ankle Clin 2002; 7: 501-513.

[18] Shrier I, Matheson GO, Kohl HW, 3rd. Achilles tendonitis: are corticosteroid injections useful or harmful? Clin J Sport Med 1996; 6: 245-250.

[19] Stein C, Hassan AH, Przewlocki R, et al. Opioids from immunocytes interact with receptors on sensory nerves to inhibit nociception in inflammation. Proc Natl Acad Sci U S A 1990; 87: 5935-5939.

[20] Muittari PA, Nelimarkka O, Seppala T, et al. Comparison of the analgesic effects of intrabursal oxycodone and bupivacaine after acromioplasty. J Clin Anesth 1999; 11: 11-16.

[21] Fellahi JL, Magues JP, Marquer B, et al. Analgesia with intraarticular injection of buprenorphine after surgery of the shoulder. Cah Anesthesiol 1995; 43: 313-318.

[22] Doss NW, Splain SH, Crimi T, et al. Intra-articular morphine, ropivacaine, and morphine/ropivacaine for pain control after arthroscopy: preliminary observation. Proc West Pharmacol Soc 2001; 44: 195-196.

[23] Stein C, Comisel K, Haimerl E, et al. Analgesic effect of intraarticular morphine after arthroscopic knee surgery. $N$ Engl J Med 1991; 325: 1123-1126.

[24] Puehler W, Zollner C, Brack A, et al. Rapid upregulation of mu opioid receptor mRNA in dorsal root ganglia in response to peripheral inflammation depends on neuronal conduction. Neuroscience 2004; 129: 473-479.

[25] Hanesch U, Heppelmann B, Messlinger K, et al. Nociception in normal and arthritic joints: Structural and functional aspects In: JWD Willis, ed. Hyperalgesia and Allodynia. New York: Raven Press; 1992: pp. 81-106.
[26] Raffa RB, Friderichs E, Reimann W, et al. Opioid and nonopioid components independently contribute to the mechanism of action of tramadol, an 'atypical' opioid analgesic. J Pharmacol Exp Ther 1992; 260: 275-285.

[27] Wordliczek J, Banach M, Garlicki J, et al. Influence of preor intraoperational use of tramadol (preemptive or preventive analgesia) on tramadol requirement in the early postoperative period. Pol J Pharmacol 2002; 54: 693-697.

[28] Likar R, Masthiasch K, Buerscher M, et al. Randomizeddouble blind, comparative study of morphine and tramado administered intraarticulary for postoperative analgesia following arthroscopic surgery. Clin Drug Invest 1995; 10: 1721.

[29] Lamplot JD, Wagner ER, Manning DW. Multimodal pain management in total knee arthroplasty: a prospective randomized controlled trial. J Arthroplasty 2014; 29(2): 329-334.

[30] Smink AJ, Bierma-Zeinstra SM, Dekker J, et al. Agreement of general practitioners with the guideline-based stepped-care strategy for patients with osteoarthritis of the hip or knee: a cross-sectional study. BMC Fam Pract 2013; 14: 33.

[31] Hosseini H, Abrisham SM, Jomeh H, et al. The comparison of intraarticular morphine-bupivacaine and tramadolbupivacaine in postoperative analgesia after arthroscopic anterior cruciate ligament reconstruction. Knee Surg Sports Traumatol Arthrosc 2012; 20(9): 1839-1844.

[32] Sipahi A, Satilmis T, Basa S. Comparative study in patients with symptomatic internal derangements of the temporomandibular joint: analgesic outcomes of arthrocentesis with or without intra-articular morphine and tramadol. $\mathrm{Br} J$ Oral Maxillofac Surg 2015; 53(4): 316-320.

[33] Joshi GP McCarroll SM, Cooney CM, et al. Intra-articular morphine for pain relief after knee arthroscopy. J Bone Joint Surg Br 1992; 74: 749-751.

[34] O'Kane JW, Jackins S, Sidles JA, et al. Simple home program for frozen shoulder to improve patients' assessment of shoulder function and health status. J Am Board Fam Pract 1999; 12: 270-277.

[35] Kivimaki J, Pohjolainen T, Malmivaara A, et al. Manipulation under anesthesia with home exercises versus home exercises alone in the treatment of frozen shoulder: a randomized, controlled trial with 125 patients. J Shoulder Elbow Surg 2007; 16: $722-726$. 